BULLETIN (New Series) OF THE

AMERICAN MATHEMATICAL SOCIETY

Volume 41, Number 3, Pages 275-278

S 0273-0979(04)01023-7

Article electronically published on April 13, 2004

\title{
APPLICATION OF HYPERBOLIC DYNAMICS TO PHYSICS: SOME PROBLEMS AND CONJECTURES
}

\author{
DAVID RUELLE
}

René Thom in memoriam.

\begin{abstract}
The long time behavior of smooth dynamical systems is, in good cases, given by an SRB measure $\rho$. The measure $\rho$ is expected often to have very discontinuous dependence on parameters of the dynamical system. This is a very unsatisfactory situation for physical applications, where one would like to differentiate $\rho$ with respect to parameters. Here we propose a solution to this difficulty, based on analytic continuation in a frequency variable $\omega$.
\end{abstract}

René Thom was a philosopher and (among other things but most successfully) a lover of that part of philosophy which we call mathematics. (I use here the term philosophy with its old meaning, which some contemporary philosophers and mathematicians might not like.) Inside and outside mathematics, Thom's interest was turned to forms and ideas. His passion was to understand geometrically the nature of things, and for this he used mathematical proofs. But he was not a formalist, and, for him, proofs remained secondary to the conceptual landscape that they revealed. One area where he invested a lot of effort was the study of differentiable dynamical systems. This smooth dynamics is where geometric forms come closest to life. I attended the regular seminar that he ran on the subject. Under the influence of Thom, the IHES was an important center for the development of differentiable dynamical systems, especially hyperbolic dynamics, in the golden years of the 1960's and 1970's. Steve Smale and his friends were frequent visitors (and the Russians came when they could).

The big question in smooth dynamics is: "What do general smooth dynamical systems look like?" What one wants is an understanding of a large set of dynamical systems. This is also a significant question for physical applications. More specifically, one would like to understand the long time behavior for typical time evolutions and how this changes when the parameters controlling the time evolution are changed.

The uniformly hyperbolic systems studied by Anosov, Smale, Thom and many others form a large set, and Smale had conjectured that they might be dense among all dynamical systems. But this is not the case, and it may be too much to hope for a very large set of dynamical systems that are understood in great detail like the uniformly hyperbolic systems. From a practical point of view, the numerical study of dynamical systems often reveals extremely long characteristic times, which

Received by the editors October 1, 2003.

2000 Mathematics Subject Classification. Primary 37C40; Secondary 37D45.

Key words and phrases. Smooth dynamics, SRB states.

(C)2004 American Mathematical Society 
make the system effectively unanalyzable (for instance the existence Lebesgue a.e. of ergodic limits remains uncertain). This is of course terrible if one wants to use the results of smooth dynamics to construct a physical theory. I shall come back to this problem, but let me mention nonequilibrium statistical mechanics as a physical problem for which smooth dynamics appears to be relevant (and on which I am currently working).

Since the early period of uniformly hyperbolic dynamical systems, perspectives have changed somewhat. In particular, a purely geometric approach has been replaced in part by an ergodic approach. Taking the system defined by a diffeomorphism $f$ of the compact manifold $M$, there are in general no $f$-invariant measures absolutely continuous with respect to Lebesgue, but there may be a measure $\rho$ such that, for $x$ in a set of Lebesgue measure $>0$,

$$
\lim _{n \rightarrow \infty} \frac{1}{n} \sum_{k=0}^{n-1} \phi\left(f^{k} x\right)=\int \rho(d y) \phi(y) .
$$

Such measures $\rho$ have come to be called SRB measures. They were first studied for uniformly hyperbolic systems (by Sinai, Ruelle, Bowen): there is exactly one SRB measure on a transitive hyperbolic attractor. The advent of Pesin theory permitted an extension of the concept of SRB measures to general diffeomorphisms (by Strelcyn, Ledrappier, Young): they are characterized by equality of the entropy $h(\rho)$ (KS invariant) with the sum of the positive Lyapunov exponents $\lambda_{i}(\rho)$. (Lyapunov exponents are defined so that if $u \in T_{x} M$, then $\left\|T_{x} f^{k} u\right\| \approx e^{\lambda_{i}}\|u\|$ for $\rho$-almost all $x$ ). Note that in the general case the set of SRB measures may be empty. J. Palis has made general conjectures on what a really large set of smooth dynamical systems might look like (they would in particular have SRB measures).

The inverse of a Lyapunov exponent is one of the characteristic times associated with a dynamical system (with ergodic measure $\rho$ ). Another characteristic time is the inverse of the rate of decay of correlations, i.e., of the rate of exponential decrease of $k \mapsto \rho\left(A\right.$. $\left.\left(B \circ f^{k}\right)\right)-\rho(A) \rho(B)$ for $|k| \rightarrow \infty$ when $A$ and $B$ are smooth functions on $M$.

An important extension of uniform hyperbolicity is provided by Hénon-like diffeomorphisms [2], [7], [8]. These diffeomorphisms cause some kind of folding in the manifold $M$, which results in nonuniform hyperbolicity. Hénon-like diffeomorphisms have SRB measures, with nonzero Lyapunov exponents, and (as shown in 8]) nonzero rate of decay of correlations. These diffeomorphisms are related to unimodal maps of the interval [0,1], like the map $x \mapsto a x(1-x)$ first studied for $a$ close to 4 by Iakobson. (There is a set $S \subset(4-\epsilon, 4)$ such that for $a \in S$ the map $x \mapsto a x(1-x)$ has an ergodic measure $\rho$ absolutely continuous with respect to Lebesgue on $[0,1]$ and with $\lambda(\rho)>0$. The set $S$ has dense complement but Lebesgue meas. $(S)>0$ ). Hénon-like diffeomorphisms have nice properties (like those of uniformly hyperbolic diffeomorphisms), but their behavior depends discontinuously on parameters (like the properties of $x \mapsto a x(1-x)$ depend discontinuously on $a$ ).

Consider now a physical problem where the long time behavior is given by an SRB measure $\rho$ for the diffeomorphism $f$. Of particular importance is how measured quantities $\rho(A)=\int \rho(d x) A(x)$ depend on parameters, i.e., on changes $\delta f$ of $f$. Introducing the vector field $X=\delta f \circ f^{-1}$, we obtain by an easy formal calculation, 
for smooth $A$,

$$
\text { * } \quad \delta \rho(A)=\sum_{k=0}^{\infty} \int \rho(d x) X(x) \cdot \nabla_{x}\left(A \circ f^{k}\right) .
$$

This formula can be justified in the uniformly hyperbolic case [6] 1] However, we expect $(*)$ to fail in the Hénon-like case because $\rho(A)$ depends discontinuously on parameters. Indeed the series $(*)$ appears to be exponentially divergent rather than convergent.

Still, $\delta \rho(A)$ should make sense physically under more general conditions than uniform hyperbolicity, and $(*)$ is the only reasonable formula one can write. How can that be? Physical situations of nonequilibrium statistical mechanics involve many degrees of freedom (and thus, high dimension). One can expect physically that in such situations the discontinuities of $\rho(A)$ as a function of parameters become invisible because they occupy a set of small measure in parameter space. How can this be reconciled with the divergence of $(*)$ ? Consider the response function of the system to a time-dependent sinusoidal force:

$$
\delta_{\omega} \rho(A)=\sum_{k=0}^{\infty} e^{i k \omega} \int \rho(d x) X(x) \cdot \nabla_{x}\left(A \circ f^{k}\right) .
$$

In good cases this quantity depends meromorphically on $\omega$ in a certain region, with poles related to discrete eigenvalues of suitable transfer operators ([5, 4], [1]), and one can hope that $(* *)$ has a well-defined value at $\omega=0$. If the poles that make (*) divergent have small residues, they could be ignored in a physical theory.

This suggests that there is an interesting class of dynamical systems for which $(* *)$ is a meromorphic function of $\omega$ in a domain containing 0 , and $\delta_{0} \rho(A)$ defines in some sense a derivative of $\rho(A)$ with respect to parameters. This is true for uniformly hyperbolic diffeomorphisms, but not known for Hénon-like diffeomorphisms, and even the case of unimodal maps is currently open.2

At this point I am tempted to adopt the attitude of René Thom, who made, in some cases, deliberately hazy mathematical statements (and obviously relished it). Here our central question is not mathematically well posed but is potentially important: can one make sense of $(*)$ in situations of physical interest? In the present note I have sketched an approach to this question, using a meromorphic extension of $(* *)$. In particular this suggests obvious conjectures for unimodal maps and Hénon-like diffeomorphisms. My hope is that the proof or disproof of these conjectures will help answer the original, somewhat imprecise, physical question.

\section{REFERENCES}

1. V. Baladi. Positive transfer operators and decay of correlations. World Scientific, River Edge, NJ, 2000. MR 2001k:37035

2. M. Benedicks and L. Carleson. "The dynamics of the Hénon map." Annals of Math. 133, 73-169 (1991). MR 92d:58116

3. D. Dolgopyat. "On differentiability of SRB states." Preprint.

4. W. Parry and M. Pollicott. Zeta functions and the periodic orbit structure of hyperbolic dynamics. Astérisque 187-188, Soc. Math. de France, Paris, 1990. MR 92f:58141

\footnotetext{
${ }^{1}$ I am indebted to D. Dolgopyat and M. Jiang for constructive criticism of the first proof of this result; see also [3].

${ }^{2}$ I am indebted to L.-S. Young, M. Benedicks, V. Baladi and M. Viana for discussions of this question.
} 
5. D. Ruelle. Thermodynamic formalism. Addison-Wesley, Reading (Mass.), 1978. MR 80g:82017

6. D. Ruelle. "Differentiation of SRB states." Commun. Math. Phys. 187, 227-241 (1997); "Correction and complements." Commun. Math. Phys. 234, 185-190 (2003). MR 98k:58144

7. M. Viana. "Strange attractors in higher dimensions." Bull. Braz. Math. Soc. 24, 13-62 (1993). MR 94k:58093

8. Q.D. Wang and L.-S. Young. "Strange attractors with one direction of instability." Commun. Math. Phys. 218, 1-97 (2001); "Strange attractors with one direction of instability in n-dimensional spaces." Preprint 2001. MR 2002m:37050

Department of Mathematics, Rutgers University, 110 Frelinghuysen Road, PiscatAWAY, NEW JERSEY 08854-8019

E-mail address: ruelle@ihes.fr

Current address: IHES, 91440 Bures sur Yvette, France 